\title{
Image denoising algorithm via spatially adaptive bilateral filtering
}

\author{
Qi Min', a , Zhou Zuofeng ${ }^{2, b}$, Liu Jing ${ }^{3, c}$, Cao Jianzhong, ${ }^{2, d}$, Wang Hao, ${ }^{2, e}$ Yan \\ Aqi $^{2, f}$, Wu Dengshan ${ }^{2, g}$, Zhang Hui ${ }^{2, h}$ and Tang Linao ${ }^{3, i}$ \\ ${ }^{1}$ The 63870 Unit of People's Liberation Army, Weinan, P.R. China, 714200 \\ ${ }^{2}$ Xi'an Institutes of Optics and Precision Mechanics, Chinese Academy of Sciences,
}

Xi'an, P.R. China, 710119

${ }^{3}$ Huawei Technologies Co., Ltd, Shenzhen, P.R. China, 518129

aever817@126.com, bfzhou@opt.ac.cn, ${ }^{\mathrm{c} l i u j i n g @ 163 . c o m, ~}{ }^{\mathrm{d}} \mathrm{cjz} @ o p t . a c . c n,{ }^{\mathrm{e}}$ wanghao@opt.ac.cn, fyanaqi@opt.ac.cn, ${ }^{9}$ dengshanwu@opt.ac.cn, 'zhanghui@163.com, 'tanglinao@163.com,

Keywords: Image denoising; Bilateral filtering; Spatially Adaptive; Local statistics characteristic

\begin{abstract}
The classical bilateral filtering algorithm is a non-linear and non-iterative image denoising method in spatial domain which utilizes the spatial information and the intensity information between a point and its neighbors to smooth the noisy images while preserving edges well. To further improve the image denoising performance, a spatially adaptive bilateral filtering image deonoising algorithm with low computational complexity is proposed. The proposed algorithm takes advantage of the local statistics characteristic of the image signal to better preserve the edges or textures while suppressing the noise. Experiment results show that the proposed image denoising algorithm achieves better performance than the classical bilateral filtering image denoising method.
\end{abstract}

\section{Introduction}

During the CCD and CMOS-based digital imaging process, the noise effect is inevitable and the first step in most image pre-processing and computer vision task is often to suppress noise while preserving the original image signal from the observed noisy image [1]. Considering the computational complexity and real-time image processing requirement, a great deal of researches about spatial domain image denoising algorithms has been done. Among the various spatial domain image denoising algorithms, the bilateral filtering method [2] can achieve good performance and of low complexity.

In conventional linear low-pass spatial domain filtering algorithms, the values of the weight are uniform or decreasing with distance from its center which means that only the spatial information is considered in the filtering process. The classical bilateral filtering method [2], takes into account both the spatial information and the intensity information between a point and its neighboring points. Moreover, the classical bilateral filtering is non-iterative and non-linear filtering process with only a single pass which make it of low complexity. Due to its simple implementation and effectiveness, the classical bilateral filtering has been widely used in image denoising, edge detection and etc.

Recently, many researchers [3 5] have devoted to the modification and improvement of the classical bilateral filtering algorithm. In this paper, a spatially adaptive bilateral filtering image deonoising algorithm with low computational complexity is proposed. The proposed method can further improve the image denoising performance by taking advantage of the local statistics characteristic of an image signal to better preserve the edges or textures while suppress the noise. Experiment results show that the proposed image denoising algorithm achieves better performance than the classical bilateral filtering image denoising method.

\section{Classical Bilateral Filtering Algorithm}

Combined spatial domain and intensity domain filtering, the classical bilateral filtering algorithm replaces the pixel values at location $\mathbf{x}$ with a weighted sum of the pixel values in a local 
neighborhood. Assume an image $f(\mathbf{x})$ is corrupted by the additive stationary white Gaussian noise $\mathbf{n}$ with zero mean and variance $\sigma_{n}^{2}$. The observed image is represented in spatial domain by $I(\mathbf{x})=f(\mathbf{x})+\mathbf{n}$.Mathematically; the output of classical bilateral filtering, which use the Gaussian functions of the Euclidean distance as the spatial closeness function and the intensity similarity function, can be defined as follows:

$$
\tilde{f}(\mathbf{x})=\frac{1}{C} \sum_{\xi \in \mathbf{N}} W_{s}(\mathbf{x}, \xi) W_{I}(\mathbf{x}, \xi) I(\xi)
$$

where the spatial weight $W_{s}(\mathbf{x}, \xi)=\exp \left\{-\|\xi-\mathbf{x}\|^{2} / 2 \sigma_{s}^{2}\right\}$, the intensity weight $W_{I}(\mathbf{x}, \xi)=\exp \left\{-|I(\xi)-I(\mathbf{x})|^{2} / 2 \sigma_{I}^{2}\right\}$ and constant $C=\sum W_{s}(\mathbf{x}, \xi) W_{I}(\mathbf{x}, \xi), \sigma_{s}$ and $\sigma_{I}$ control the tradeoff of the weights in spatial domain and intensity domain, respectively.

\section{The Proposed Algorithm}

The main advantage of the classical bilateral filtering method is that it allows for considering both the spatial locality and neighboring points with similar amplitudes at the same time which make it can better preserving the image edges and textures than the conventional linear filtering algorithms. From Eq.1, it can be seen that the behavior of the classical bilateral filtering method is highly depending on the selection of the two parameters $\left\{\sigma_{s}, \sigma_{I}\right\}$. But these two parameters, which control the spatial weight and intensity weight, are unchanged for the entire image signal during the filtering process. In other words, the local filters used in classical bilateral filtering method is changing depend on the underlying image signal. But the changing degree is constrained by a fixed set of parameters $\left\{\sigma_{s}, \sigma_{I}\right\}$. Unfortunately, as far as I know, there is not much theoretical research on how to select the optimal values for $\left\{\sigma_{s}, \sigma_{I}\right\}$ in the filtering process. Therefore, the two parameters are usually selected empirically. In [3], the authors analyze the two parameters as a function of noise variance for image denoising applications and give two conclusions about selecting the two parameters: one is that a good choice for the value $\sigma_{s}$ is roughly $[1.5 \sim 2.1]$; the other is that, under the mean square error sense, the optimal value of $\sigma_{I}$ is linearly proportional to the standard deviation of the noise. But once the two parameters are determined, they will also un-change in the filtering process for the entire image signal.

The natural image is a typically non-stationary signal which consists of smooth, edge and texture regions. Different regions have different statistics characteristics. Ideally, to achieve the best image filtering performance, the optimal values of $\left\{\sigma_{s}, \sigma_{I}\right\}$ or the optimal weights should be the one that changes as the local statistics characteristics of the natural image change. Therefore, in this paper, we propose the spatially adaptive bilateral filtering method to make the values of $\left\{\sigma_{s}, \sigma_{I}\right\}$ change as the spatial location change in the filtering process. The spatially adaptive bilateral filtering method is represented as follows:

$$
\hat{f}(\mathbf{x})=\frac{1}{\hat{C}} \sum_{\xi \in \mathbf{N}} W_{s}(\mathbf{x}, \xi) \hat{W}_{I}\left(\mathbf{x}, \xi, \sigma_{n}\right) I(\xi)
$$

where the new intensity weight $\hat{W}_{I}\left(\mathbf{x}, \xi, \sigma_{n}\right)=\exp \left\{-\alpha \tilde{\sigma}_{s}|I(\xi)-I(\mathbf{x})|^{2} / \sigma_{n}^{2}\right\}$,the new normalization constant $\hat{C}=\sum_{\xi \in \mathbf{N}} W_{s}(\mathbf{x}, \xi) \hat{W}_{I}(\mathbf{x}, \xi)$,the parameter $\alpha, \sigma_{n}^{2}$ and $\tilde{\sigma}_{s}$ represent a small constant, the noise variance and the local standard deviations of the image signal, respectively. 
The intensity parameter $\sigma_{I}$ in classical bilateral filtering plays a crucial role in the filtering process. Given the spatial parameter $\sigma_{s}$, larger the parameter $\sigma_{I}$ will lead the Gaussian shape of intensity weight more flatness and smaller the parameter $\sigma_{I}$ will lead the shape of intensity weight more sharpness. For the natural image signal, in the smooth regions, one could expect the shape of intensity weight more flatness which can effectively smooth the noise; vice versa, in the edge and texture regions, one could expect the shape of intensity weight more sharpness which can preserve the edges and textures well. Beyond the adaptation of the local characteristics of the image signal, the intensity weight should also depend on the noise level. When the signal to noise ratio is low, the shape of intensity weight should be more flatness to better suppress the noise. Therefore, the optimal parameter $\sigma_{I}$ should adaptive with the local characteristics of the image signal and the noise level. Considering the computational complexity, we use the local deviation to reflect the local statistical characteristics of the image.

Comparing the new intensity weight with the classical intensity weight, it can be seen that the classical intensity parameter $\sigma_{I}$ is replaced by

$$
\tilde{\sigma}_{I}=\sigma_{n}^{2} / \alpha \tilde{\sigma}_{s} .
$$

The new intensity parameter $\tilde{\sigma}_{I}$ is determined both by the noise variance $\sigma_{n}^{2}$ and the local standard deviation $\tilde{\sigma}_{s}$ of the image signal. From Eq.2, it can be seen that the local standard deviation $\tilde{\sigma}_{s}$ of the image signal is considered while the intensity parameter in [3] is not. Thus, the new intensity weight $\hat{W}_{I}(\mathbf{x}, \xi)$ will change as the local statistical characteristics change. Actually, the local standard deviation $\tilde{\sigma}_{s}$ can be seen as a rough classifier of the image signal. When the reference point locates in the smooth regions, the local standard deviation is relative small. Thus, the shape of the intensity weight is more flatness which makes the filter can effectively remove the noise; vice versa, for the edge or texture regions in an image, the local standard deviation is relative large. The shape of the intensity weight is more sharpness which makes the filter can preserve the edges and textures well. Beyond the local standard deviation of the image signal, there is a constant $\alpha$ which needs to be pre-determined. In this paper, we empirically set this parameter to be 0.003 .

\section{Experiment Results}

In order to evaluate the denoising performance, we test the proposed algorithm on several 8-bit gray images. The noisy images were degraded by using Eq.1 with noise standard deviations20, 30 and 40. Based on the discussion in the previous sections and the parameter selection strategy used in [3], we chose the parameters for the spatially adaptive bilateral filtering method as follows: the spatial parameter $\sigma_{s}=1.8$, the size of the squared-shaped window which is used for the filtering process and the estimation for the local standard deviation of the image signal is $7 \times 7$.The output PSNR of the proposed algorithm and the classical bilateral filtering method are listed in Table 1.

Table 1 the output PSNR of different image denoising algorithms

\begin{tabular}{|l|l|l|l|l|l|l|}
\hline \multirow{2}{*}{$\sigma_{n}$} & 20 & \multicolumn{2}{l|}{30} & \multicolumn{2}{l|}{40} \\
\cline { 2 - 7 } & BF [2] & Proposed & BF[2] & Proposed & BF[2] & Proposed \\
\hline Boat & 27.20 & $\mathbf{2 7 . 3 1}$ & 25.07 & $\mathbf{2 5 . 4 5}$ & 23.63 & $\mathbf{2 4 . 2 1}$ \\
\hline Goldhill & 28.45 & $\mathbf{2 8 . 9 1}$ & 26.72 & $\mathbf{2 7 . 0 8}$ & 24.58 & $\mathbf{2 5 . 6 8}$ \\
\hline Peppers & 28.82 & $\mathbf{2 9 . 3 0}$ & 26.76 & $\mathbf{2 7 . 7 9}$ & 25.10 & $\mathbf{2 6 . 5 0}$ \\
\hline House & 28.91 & $\mathbf{2 9 . 5 6}$ & 26.62 & $\mathbf{2 7 . 4 3}$ & 24.36 & $\mathbf{2 5 . 4 5}$ \\
\hline Lena & 29.62 & $\mathbf{3 0 . 5 1}$ & 28.13 & $\mathbf{2 8 . 4 0}$ & 25.22 & $\mathbf{2 6 . 8 3}$ \\
\hline
\end{tabular}

From Table 1, it can be seen that, the proposed algorithm achieves better performance than the classical bilateral filtering method for all test images. For example, the proposed algorithm is $0.5 \mathrm{~dB}$ 
better than the classical bilateral filtering method on average with the noise standard deviation 20 . Actually, the lower of the signal to noise ratio, the more PSNR improvement of the proposed algorithm can achieved compared to the classical bilateral filtering method. In order to illustrate the visual effect, a local region of 'Lena' image is illustrated in Fig. 1. It can be seen that the proposed algorithm preserves better the texture and edge structures in Lena's hat and eye regions than the classical bilateral filtering method.

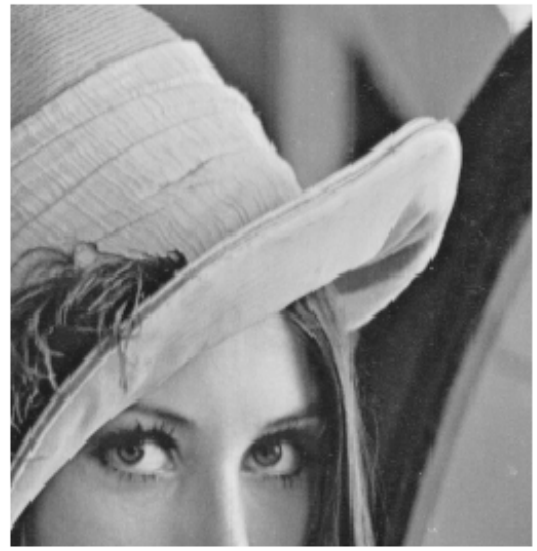

(a)

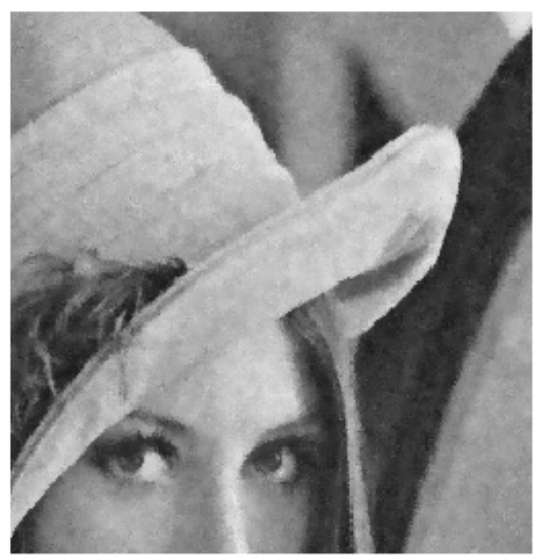

(c)

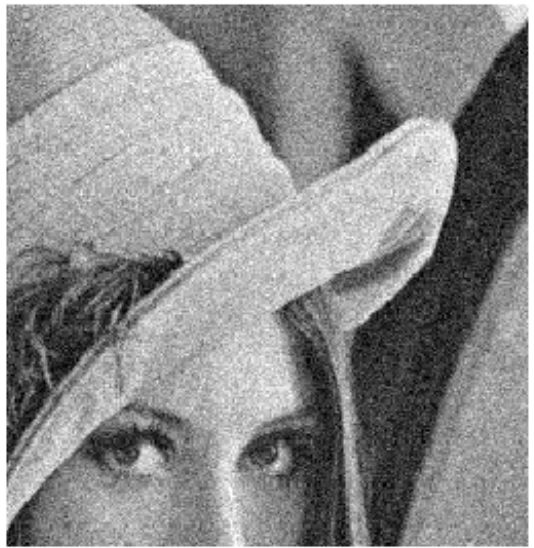

(b)

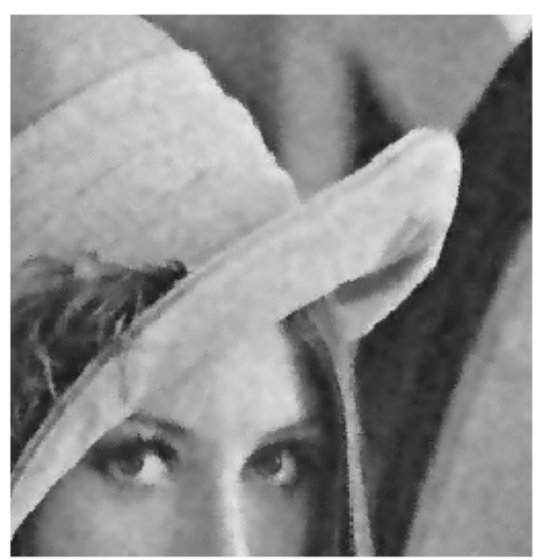

(d)

Fig.1 Zoomed regions: (a) original 'Lena', (b) noisy image $\sigma=20$, (c) denoised image in[2] (the PSNR is $29.42 \mathrm{~dB}$ ) and (d) denoised image using the proposed algorithm (the PSNR is $30.24 \mathrm{~dB}$ )

\section{Conclusions}

In this paper, a simple and effective spatially adaptive bilateral filtering image deonoising algorithm is proposed to improve the image denoising performance of the classical bilateral filtering method. Experiment results show the effectiveness of the proposed image denoising algorithm.

\section{References}

[1] R.C. Gonzalez, R.E. Woods, S.L. Eddins, Digital image processing. Prentice Hall, 2002.

[2] C. Tomasi, R.Manduchi, Bilateral filtering for gray and color images, in: Proceedings of the ICCV( 1998), p. 836.

[3] M. Zhang, B.K. Gunturk, Multiresolution Bilateral Filtering for Image Denoising, IEEE Trans. Image Process. Vol.17 (2008), p. 2324.

[4] B.K. Gunturk, Fast bilateral filter with arbitrary range and domain kernels, IEEE Trans. Image Process. Vol.20 (2011), p. 2690.

[5] A. Wong, Adaptive bilateral filtering of image signals using local phase characteristics, Signal Process. (Elsevier), Vol.88 (2008) p.1615. 